\title{
Percepção dos Pais de Pacientes com Transtorno do Espectro Autista sobre o Atendimento Odontológico com Sedação Leve à Moderada
}

Parents' Perception of Patients with Autism Spectrum Disorder about Dental Care with Mild to Moderate Sedation Percepción de los Padres de Pacientes con Trastornos del Espectro Autista Sobre el Cuidado Dental con Sedación Ligera a Moderada

Suellen Pestana Moreira Ribeiro DE LIMA Cirurgiã-Dentista pela Faculdade Uninassau, 58030-000 - João Pessoa - PB, Brasil https://orcid.org/0000-0002-0461-2798

Wandersson Marcone e Barbosa SILVA

Cirurgião-Dentista pela Faculdade Uninassau, 58030-000 - João Pessoa - PB, Brasil Herrison Félix Valeriano DA SILVA

Mestrando em Odontologia da Universidade Federal da Paraíba (UFPB) - 58051-900 João Pessoa - PB, Brasil https://orcid.org/0000-0001-6714-3151

Thauany Vasconcelos Soares DA SILVA

Mestranda em Odontologia da Universidade Federal da Paraíba (UFPB) - 58051-900 João Pessoa - PB, Brasil https://orcid.org/0000-0002-6831-9224

Gloria Maria Pimenta CABRAL

Professora Doutora, especialista em Odontologia para Pacientes com Necessidades Especiais, João Pessoa - PB, Brasil https://orcid.org/0000-0003-0104-1448

Rafaella Bastos LEITE

Professora Doutora, do curso de Odontologia da Faculdade Uninassau, 58030-000 - João Pessoa - PB, Brasil https://orcid.org/0000-0002-3304-120X

\section{Resumo}

O Transtorno do Espectro Autista (TEA) é uma alteração do desenvolvimento neurológico com início precoce, caracterizado por comprometimento das habilidades sociais e da linguagem, além de comportamentos estereotipados. Pacientes com TEA são indivíduos que representam um grande desafio para os cirurgiões-dentistas, devido às suas manifestações clínicas complexas e variadas, sendo assim, o tratamento odontológico para este grupo, muitas vezes, é considerado desafiador para os pais e para os profissionais. Esse trabalho objetivou-se avaliar a percepção dos pais de pacientes com TEA sobre o uso da sedação leve à moderada no atendimento odontológico. Foi realizado uma pesquisa com estudo descritivo e uma abordagem qualitativa para responder às questões dinâmicas e complexas propostas aos pais sobre sedação leve à moderada em pacientes com Transtorno do Espectro autista. A pesquisa foi realizada em um consultório particular, na cidade de João Pessoa, Paraíba. Os sujeitos do estudo foram pais de pacientes com autismo que tenham sido sedados ao menos uma vez em consulta odontológica. Os entrevistados demonstraram bastante satisfação com o uso da sedação no atendimento odontológico de pacientes com TEA. A pesquisa irá contribuir proporcionando uma realidade mais assertiva devido à experiência pessoal com os filhos submetidos a sedação durante atendimento odontológico.

Descritores: Transtorno do Espectro Autista; Sedação Consciente; Percepção; Assistência Odontológica para Pessoas com Deficiências.

\section{Abstract}

Autism Spectrum Disorder (ASD) is an early-onset neurological development disorder characterized by impairment of social and language skills, in addition to stereotyped behaviors. Patients with ASD are individuals who represent a great challenge for dentists, due to their complex and varied clinical manifestations, thus, dental treatment for this group is often considered challenging for parents and professionals. This study aimed to evaluate the perception of parents of patients with ASD about the use of mild to moderate sedation in dental care. A research with descriptive study and a qualitative approach was carried out to answer the dynamic and complex questions proposed to parents about mild to moderate sedation in patients with Autistic Spectrum Disorder. The research was carried out in a private practice in the city of João Pessoa, Paraíba. The study subjects were parents of patients with autism who had been sedated at least once in a dental appointment. Respondents showed great satisfaction with the use of sedation in dental care for patients with ASD. The research will contribute by providing a more assertive reality due to the personal experience with children undergoing sedation during dental care.

Descriptors: Autism Spectrum Disorder; Conscious Sedation; Perception; Dental Care for Disabled.

\section{Resumen}

El trastorno del espectro autista (TEA) es un trastorno del desarrollo neurológico de inicio temprano que se caracteriza por el deterioro de las habilidades sociales y del lenguaje, además de comportamientos estereotipados. Los pacientes con TEA son individuos que representan un gran desafío para los odontólogos, debido a sus complejas y variadas manifestaciones clínicas, por lo que el tratamiento odontológico para este grupo suele ser considerado un desafío para los padres y profesionales. Este estudio tuvo como objetivo evaluar la percepción de los padres de pacientes con TEA sobre el uso de sedación leve a moderada en el cuidado dental Se realizó un estudio descriptivo con abordaje cualitativo para dar respuesta a las preguntas dinámicas y complejas propuestas a los padres sobre la sedación leve moderada en pacientes con Trastorno del Espectro Autista. La investigación se llevó a cabo en un consultorio privado de la ciudad de João Pessoa, Paraíba. Los sujetos del estudio eran padres de pacientes con autismo que habían sido sedados al menos una vez en una cita odontológica, quienes mostraron gran satisfacción con el uso de la sedación en la atención odontológica de pacientes con TEA. La investigación contribuirá a brindar una realidad más asertiva debido a la experiencia personal con niños sometidos a sedación durante el cuidado dental.

Descriptores: Trastorno del Espectro Autista; Sedación Consciente; Percepción; Atención Dental para Personas con Discapacidad.

INTRODUÇÃO

O vocábulo "autismo" passou por diversas alterações com o passar do tempo, e atualmente é caracterizado como Transtorno do Espectro Autista (TEA) pelo Manual Diagnóstico e Estatístico de Transtornos Mentais (DSM-V) ${ }^{1}$.
As características do espectro apresentam prejuízos na comunicação e/ou interação social, bem como nos comportamentos que podem incluir os interesses e os padrões de atividades, sinais 
que estão presentes desde a infância e podem limitar ou prejudicar o funcionamento diário do indivíduo ${ }^{1}$. O TEA é uma alteração do desenvolvimento neurológico com início precoce, caracterizado por comprometimento das habilidades sociais e da linguagem, além de comportamentos estereotipados ${ }^{2}$, porém sua etiologia ainda não foi totalmente elucidada. Alguns estudos disponíveis apontam para uma combinação de fatores genéticos e ambientais ${ }^{3}$.

Em relação ao diagnóstico para 0 Transtorno do Espectro Autista, a observação comportamental da criança é a mais utilizada. Sendo o diagnóstico clínico baseado principalmente na presença de distúrbios de interação social, interesses restritos, padrões estereotipados do comportamento e distúrbios de comunicação. Apesar da evolução farmacológica, ainda não existe nenhuma medicação específica ou tratamento para a cura do TEA, contudo, ainda que não haja modificação comportamental total, o auxílio farmacológico atenua sintomas específicos exacerbados, fármacos estabilizadores de humor, anticonvulsivantes, antipsicóticos e antidepressivos. Vale salientar que muitos pacientes não necessitam desses medicamentos e é de extrema importância o estímulo precoce para que haja uma atenuação de possíveis sintomas futuros ${ }^{4}$.

Como o TEA não tem cura, a busca pelo tratamento específico porta consigo, entretanto, a importância de atenuar os déficits apresentados, pois, alguns tratamentos podem ser mais eficazes para uns e menos para outros, em função de cada autista apresentar um nível de desenvolvimento diferente do outro. Contudo, no que se refere ao tratamento, ainda a psicoterapia comportamental é a mais preconizada juntamente com o processo de condicionamento que facilita os cuidados com o autista, tornando-o mais estruturado emocionalmente e organizado ${ }^{5}$.

Várias características presentes em pessoas com TEA têm relevância na rotina do tratamento odontológico, tais como, sensibilidade sensorial exacerbada, respostas extremas e peculiares a sons específicos, aromas, toques ou texturas, que podem levar o paciente a tentativa de fuga e comportamento agressivo, e à hipossensibilidade ou indiferença à dor ${ }^{1}$. Entretanto, é fundamental que a criança autista apresente sempre adequada saúde bucal e, para isso, é necessário que haja uma prevenção. Normalmente, o primeiro contato da criança autista com 0 dentista acontece tardiamente, e isso torna o atendimento ainda mais complexo. Ganhar a confiança do autista requer tempo e, geralmente, não se consegue êxito na primeira consulta ${ }^{6}$.

Orellana et al. ${ }^{7}$ referem que os pacientes com TEA são os indivíduos com necessidades especiais que representam um grande desafio para os cirurgiões-dentistas, devido às suas manifestações clínicas complexas e variadas, sendo assim, o tratamento odontológico para este grupo, muitas vezes, é considerado desafiador para os pais e para os profissionais. Dificuldade de abordagem, comportamento repetitivo e limitado e recusa para responder aos comandos são alguns dos desafios encontrados ${ }^{6}$.

Paralelamente, as crianças com TEA frequentemente cooperam pouco com os procedimentos médicos, particularmente aqueles considerados invasivos, como atendimento odontológico ${ }^{8}$.

As necessidades odontológicas do paciente autista são semelhantes com as de outros pacientes, porém pode ser mais difícil proporcionar um tratamento odontológico eficaz.

Muitas pessoas com TEA apresentam inflexibilidade à rotina e ao meio ambiente, ou seja, sentar numa sala de espera desconhecida pode resultar em ansiedade e agitação não cooperando com o atendimento odontológico ${ }^{3}$.

Segundo Orellana et al. $^{7}$ em seus estudos, foi possível observar que $60-90 \%$ dos pacientes com TEA têm um perfil sensorial incomum, incluindo disfunção no registro de sensibilidade oral. Com isso, o consultório odontológico, expressa um lugar de estímulo de ansiedade com luzes fluorescentes fortes, equipamentos que geram ruídos agudos como a caneta de alta rotação, além de materiais de textura, gosto e aroma desconhecidos ${ }^{9}$.

Em pacientes autistas não colaborativos, na qual muitas tentativas de abordagem e atendimento já foram tentadas, a sedação tornase uma opção interessante ${ }^{6}$. Nesse contexto, a sedação medicamentosa pode representar uma opção viável para a realização do tratamento odontológico em pacientes com TEA ${ }^{10}$.

Conforme Polli et al. ${ }^{11}$ a sedação, leve ou moderada, obtida através da medicação oral, pode representar uma boa opção para o tratamento odontológico de pacientes não colaboradores, desde que sejam seguros e efetivos para conter o paciente.

Diante do exposto, é notório que a sedação em consultório odontológico parece ser benéfica e eficaz em pacientes com autismo. Os pais dos pacientes com TEA possuem conhecimento sobre os benefícios da sedação no atendimento odontológico? É de suma importância salientar os pais sobre esses 
benefícios, além de possuir mínimo risco à saúde e à vida do paciente, o que proporciona segurança ao seu uso, promovendo a realização de um atendimento odontológico confortável e com tranquilidade.

Essa pesquisa tem como objetivo, avaliar a percepção dos pais de pacientes com TEA sobre o uso da sedação leve à moderada no atendimento odontológico, investigar o nível de conhecimento dos pais sobre o uso de sedação em pacientes com TEA,explorar as percepções experimentadas pelos pais sobre 0 uso de sedação em pacientes com TEA, salientar os pais de pacientes com TEA sobre os benefícios da sedação no atendimento odontológico e enfatizar a eficácia e segurança da técnica de sedação em pacientes com TEA.

MATERIAL E MÉTODO

Trata-se de um estudo descritivo e uma abordagem qualitativa para responder às questões dinâmicas e complexas propostas aos pais sobre sedação leve à moderada em pacientes com Transtorno do Espectro Autista, tendo em vista que a pesquisa qualitativa responde a questões muito particulares e é importante para compreender as relações que se dão entre pacientes, pais, profissionais e sua situação.Foi explicado o objetivo, o método, a garantia de confidencialidade dos dados e da possibilidade de desistência em qualquer etapa da pesquisa. Os pais foram convidados a participar e após explicação sobre riscos e benefícios do estudo, foram solicitadas assinaturas do Termo de Consentimento Livre e Esclarecido. A técnica utilizada para coleta de dados foi a entrevista semi estruturada, ou seja, com estrutura previamente definida, mas que possibilita ao entrevistado discorrer livremente sobre o tema, sem a interferência do pesquisador. Para avaliação do instrumento proposto, foi realizado um projeto-piloto com os pais e as entrevistas foram gravadas e depois transcritas para serem analisadas. Partindo dessas considerações, o tema proposto foi apresentado a partir de dados empíricos relativos a depoimentos selecionados do conjunto de dados dos participantes do estudo e analisados com apoio da literatura.

Os participantes do estudo foram mães e pais de pacientes com autismo que foram sedados ao menos uma vez em consulta odontológica. A seleção da amostra foi intencional, foram convidados para entrar no estudo pais que estavam acompanhando a consulta odontológica.Sendo uma amostra de 6 entrevistados, incluindo mães e pais, pois, a partir da quinta entrevista, alcançou-se a saturação dos dados. Como critério de inclusão, foram escolhidos pacientes diagnosticados com Transtorno do Espectro Autista submetidos à sedação leve ou moderada em consulta odontológica, ao menos uma vez. Como critério de exclusão, foram escolhidos pacientes com TEA que já estejam colaborativos à consulta odontológica.

A técnica utilizada para coleta de dados foi entrevista semiestruturada, através de gravação por meio de aparelho celular. Após a leitura das entrevistas transcritas, as mesmas foram analisadas por um único examinador, segundo a análise temática que se coloca como uma técnica de interpretação de textos. Foram coletados dados de identificação dos pais (iniciais do nome). As perguntas norteadoras da entrevista semiestruturada abordaram a percepção dos pais de pacientes com Autismo sobre 0 atendimento odontológico com sedação leve à moderada e sua opinião sobre como a sedação pode contribuir para um atendimento mais confortável para este paciente com necessidade especial.

RESULTADOS

Os quadros 1 a 6 apresentam a transcrição das perguntas e respostas.

Quadro 1. Pergunta sobre a idade.

\begin{tabular}{|l|l|}
\hline \multicolumn{2}{|c|}{ Qual a idade do seu filho ou filha? } \\
\hline Entrevistado 1 & "9 anos" \\
\hline Entrevistado 2 & "4 anos" \\
\hline Entrevistado 3 & "4 anos e meio" \\
\hline Entrevistado 4 & "13 anos" \\
\hline Entrevistado 5 & "11 anos" \\
\hline Entrevistado 6 & "8 anos" \\
Fonte: Dados da Pesquisa
\end{tabular}

Quadro 2. Pergunta se o(a) filho(a) já teve experiência de dor ou trauma dentário.

\begin{tabular}{|c|c|}
\hline \multicolumn{2}{|c|}{$\begin{array}{l}\text { Seu filho ou filha já teve experiência de dor ou trauma em } \\
\text { algum dente? }\end{array}$} \\
\hline Entrevistado 1 & $\begin{array}{l}\text { "Não teve, mas já visitou um dentista onde se } \\
\text { assustou, pois não houve antecipação, ela não } \\
\text { esperava que a cadeira fosse se movimentar, a } \\
\text { cadeira subiu e a dentista já chegou com o } \\
\text { motorzinho para limpar os dentes dela. Então ela } \\
\text { ficou assustada, falou que não queria voltar." }\end{array}$ \\
\hline Entrevistado 2 & $\begin{array}{l}\text { "Sim e não. Ele teve uma experiência de dor na } \\
\text { intervenção." }\end{array}$ \\
\hline Entrevistado 3 & $\begin{array}{l}\text { "Sim, ele já teve uma experiência bem ruim antes } \\
\text { da gente conhecer o processo da sedação. Hoje é a } \\
\text { paz que a gente tem em casa." }\end{array}$ \\
\hline Entrevistado 4 & "Sim, já aconteceu duas vezes." \\
\hline Entrevistado 5 & "Sim." \\
\hline Entrevistado 6 & "Sim." \\
\hline
\end{tabular}

Quadro 3. Pergunta sobre a quantidade de vezes que o paciente já foi sedado em atendimento odontológico.

\begin{tabular}{|c|c|}
\hline \multicolumn{2}{|c|}{$\begin{array}{l}\text { Quantas vezes seu filho ou filha foi sedado em atendimento } \\
\text { odontológico? }\end{array}$} \\
\hline Entrevistado 1 & "1 vez" \\
\hline Entrevistado 2 & "2 vezes" \\
\hline Entrevistado 3 & "2 vezes" \\
\hline Entrevistado 4 & "1 vezes" \\
\hline Entrevistado 5 & "3 vezes" \\
\hline Entrevistado 6 & "1 vez" \\
\hline
\end{tabular}


Quadro 4. Pergunta sobre o que achou do uso da sedação.

\begin{tabular}{|c|c|}
\hline & $\begin{array}{l}\text { O que você achou de os procedimentos } \\
\text { serem realizados com o uso da sedação? }\end{array}$ \\
\hline Entrevistado 1 & $\begin{array}{l}\text { "No início fiquei apreensiva, fiquei com medo, } \\
\text { porque eu não sabia como ia ser, o que ela ia usar, } \\
\text { não sabia como a minha filha ia ficar e depois que } \\
\text { eu vi tudo como foi feito e muito bem feito eu } \\
\text { fiquei supersatisfeita." }\end{array}$ \\
\hline Entrevistado 2 & $\begin{array}{l}\text { "Eu posso afirmar a você comparativo aí que a } \\
\text { gente viveu antes da sedação. Então ele teve } 3 \\
\text { procedimentos antes da sedação, ele tem } \\
\text { problema na polpa do dente, ele tem a má } \\
\text { formação da dentina, então ele teve que fazer uma } \\
\text { proteção dessa dentina. Foi algo absurdamente } \\
\text { traumático ao ponto dele quase desmaiar na } \\
\text { cadeira. Então, um dos } 3 \text { procedimentos que ele } \\
\text { realizou foi o mais traumático que teve que } 5 \\
\text { pessoas segurar essa criança para poder realizar o } \\
\text { procedimento. Eu segurando ele, abraçando ele, a } \\
\text { mãe, um outro dentista, e outros } 2 \text { profissionais } \\
\text { fazendo a intervenção. Ele gritava } \\
\text { desesperadamente, uma coisa traumática, que } \\
\text { quando ele saiu de lá, saiu com o corpo mole, } \\
\text { semi-desacordado. Demorou e isso causou um } \\
\text { trauma subsequente, o de ele associar todo mundo } \\
\text { de branco a esse procedimento. Ou seja, teve uma } \\
\text { extensão do trauma que complicou todas terapias } \\
\text { que ele fazia, até hoje quando ele vê uma pessoa } \\
\text { de branco, ele fica com o pé atrás. Já no } \\
\text { procedimento de sedação, nada aconteceu, aquele } \\
\text { pequeno procedimento de aplicação do sedativo, } \\
\text { logo depois ele já quase desacordado, já sobre o } \\
\text { efeito de sedação e só controlar a sedação para } \\
\text { fazer a intervenção. Ele dormiu, acordou feliz e foi } \\
\text { brincar." }\end{array}$ \\
\hline Entrevistado 3 & $\begin{array}{l}\text { "Totalmente diferente, é outro nível, outro mundo. } \\
\text { Porque você sabe que a criança vai passar por um } \\
\text { procedimento difícil que para ele aquilo é o fim do } \\
\text { mundo. Se assustava, sudorese intensa, mal estar, } \\
\text { praticamente desmaiado, medo. Aquele } \\
\text { motorzinho faz medo até para adulto, imagina } \\
\text { para a criança aquela coisa de segurar, de abrir } \\
\text { boca. O que não acontece na sedação, porque a } \\
\text { sedação espera o tempo da criança, ela ficar } \\
\text { molinha, perder as forças, fora que a criança não } \\
\text { lembra o que tá passando, não fica o trauma nem } \\
\text { para ele, nem para a gente." }\end{array}$ \\
\hline Entrevistado 4 & $\begin{array}{l}\text { "Ele ficaria muito agitado, porque como ele tem o } \\
\text { espectro autista, ele fica muito agitado." }\end{array}$ \\
\hline Entrevistado 5 & $\begin{array}{l}\text { "Muito bom, porque é um tratamento difícil e a } \\
\text { sedação ajuda bastante!" }\end{array}$ \\
\hline Entrevistado 6 & "Supertranquilo e confortável." \\
\hline
\end{tabular}

Quadro 5. Pergunta sobre a contribuição da sedação para um posterior condicionamento.

\begin{tabular}{|c|c|}
\hline \multicolumn{2}{|c|}{$\begin{array}{l}\text { Você acha que o uso da sedação contribui para um posterior } \\
\text { condicionamento do seu filho(a)? Por quê? }\end{array}$} \\
\hline Entrevistado 1 & $\begin{array}{l}\text { "Sem dúvidas contribuiu e ela pede para voltar. } \\
\text { Não ficou traumas.” }\end{array}$ \\
\hline Entrevistado 2 & $\begin{array}{l}\text { "Total. Na verdade, ele não só contribui com o } \\
\text { condicionamento, mas evita um comportamento } \\
\text { problema, por ele não ter a experiência de dor e } \\
\text { não ter a lembrança do procedimento, evita todo } \\
\text { um comportamento problema que poderia ser e já } \\
\text { foi gerado na intervenção anterior, onde ele } \\
\text { guardou memória e isso se estendeu para outras } \\
\text { terapias. Realmente evita problemas." }\end{array}$ \\
\hline Entrevistado 3 & $\begin{array}{l}\text { "Sim, porque ele não vai guardar lembranças ruins. } \\
\text { Daquela outra forma, ele vai guardar e levar pro } \\
\text { resto da vida dele. Com a sedação não, quando ele } \\
\text { for crescendo a gente vai explicando, ele não vai } \\
\text { chegar com medo, ele não vai ter o trauma do } \\
\text { dentista. Eu até me arrepio em falar!" }\end{array}$ \\
\hline Entrevistado 4 & $\begin{array}{l}\text { "Pode com certeza. Para tentar depois o } \\
\text { condicionamento será bem melhor, pois não terá } \\
\text { traumas." }\end{array}$ \\
\hline Entrevistado 5 & $\begin{array}{l}\text { "Sim, porque antes quando eu levava ela no } \\
\text { dentista, sentava na cadeira e precisava de } 4 \\
\text { pessoas para segurar e conseguir fazer uns } \\
\text { procedimentos simples, porque ela não ajuda!" }\end{array}$ \\
\hline Entrevistado 6 & $\begin{array}{l}\text { "Com certeza, pois meu filho não terá lembranças } \\
\text { traumáticas, o que vai favorecer para que possamos } \\
\text { condicionar ele quanto a ida ao dentista de forma } \\
\text { tranquila, que ele passe a ver de uma forma } \\
\text { positiva." }\end{array}$ \\
\hline
\end{tabular}

Quadro 6. Pergunta se gostaria de acrescentar mais alguma contribuição.

\begin{tabular}{|l|l|}
\hline & $\begin{array}{l}\text { Tem mais alguma coisa que você gostaria } \\
\text { de contribuir? }\end{array}$ \\
\hline Entrevistado 1 & $\begin{array}{l}\text { "Vou falar como adulta. Até hoje eu não gosto de } \\
\text { dentista, eu acho que sofri muito quando era } \\
\text { criança. De me segurarem, de fazer procedimento } \\
\text { a força. Então até hoje eu não gosto de ir ao } \\
\text { dentista. e eu não queria que isso acontecesse com } \\
\text { a minha filha, então eu acho que esse tipo de } \\
\text { sedação poderia ser feito com todas as crianças, } \\
\text { tanto as neurotípicas quanto as atípicas. Eu } \\
\text { superindico." }\end{array}$ \\
\hline Entrevistado 2 & "Abençoada sejam as sedações." \\
\hline Entrevistado 3 & $\begin{array}{l}\text { "Pbrigada pela sedação existir!" } \\
\text { sentir dor, eu acho que deveria ser tudo sedado. }\end{array}$ \\
\hline Entrevistado 4 & $\begin{array}{l}\text { Eu como adulto prefiro, imagina a criança. Eu } \\
\text { acho importante." }\end{array}$ \\
\hline Entrevistado 5 & "Tudo ótimo!" \\
\hline Entrevistado 6 & $\begin{array}{l}\text { "O uso da sedação permite um atendimento mais } \\
\text { humanizado." }\end{array}$ \\
\hline Fonte: Dados da Pesquisa
\end{tabular}

DISCUSSÃO

Os dados qualitativos analisados foram transcritos e apresentados em quadros para que - leitor possa visualizar com maior facilidade a intensidade das respostas. A pesquisa foi respondida tanto por mães e pais de pacientes diagnosticados com Transtorno do Espectro Autista. Assim sendo, o quadro 1 mostrou que as crianças diagnosticadas com Transtorno do Espectro Autista cujo os pais responderam a pesquisa possuem entre 4 e 13 anos de idade.

Em relação ao quadro 2, pode-se observar que a maioria dos pacientes já tiveram experiência de dor ou trauma dentário. Dos 6 pais que responderam à pesquisa, 5 relataram que o(a) filho(a) já vivenciaram experiência de dor ou trauma em algum dente.

Conforme Araújo e Romero ${ }^{12}$, devido à dor ser definida como uma experiência subjetiva e grande parte das pessoas não saberem descrevê-la, muitos profissionais de saúde possuem dificuldade em abordar e caracterizar a dor. Assim, é importante que o profissional tenha sensibilidade para perceber a presença e saiba mensurar e caracterizar a dor, para que, assim, façam um diagnóstico correto e escolham o melhor tratamento possível.

Ainda sobre queixa de dor, Jordan ${ }^{13}$ defende que pacientes com dor, principalmente do tipo crônica, frequentemente possuem ansiedade e depressão que acabam influenciando negativamente na realização de atividades cotidianas. E quando se trata de pacientes com TEA, essa ansiedade aumenta de forma exacerbada, tendo em vista, que pacientes diagnosticados com autismo podem já apresentar um grande nível de ansiedade. Para Berkovits et al. ${ }^{14}$, em geral, as pessoas com autismo podem apresentar dificuldades de compreender suas emoções, de criar relação interpessoal e são bastante ligadas a objetos e o local onde vivem, que quando alteradas suas 
rotinas de trabalho, podem aumentar a auto agressão, ansiedade e irritação.

No quadro 3 observa-se que três pacientes foram sedados uma vez, dois foram sedados por duas vezes e um foi sedado por três vezes. Já no quadro 4 comparando as respostas dadas pelos pais entrevistados, percebe-se que houve unanimidade quando a satisfação com 0 uso da sedação em atendimento odontológico. Apenas um entrevistado relatou que sentia medo por não ter conhecimento da ação da droga, como reagiria no organismo do seu (sua) filho(a), porém quando entendeu as vantagens ficou super satisfeito.

Segundo Oliveira ${ }^{15}$ a Odontologia possuem diversos métodos utilizados para o controle e administração da ansiedade, do medo, da dor e do comportamento em PNEs e odontopediátricos. Essas técnicas incluem características do tipo psicológicas não farmacológicas (relaxamento e comportamento) e farmacológicas. Em certos casos, estes métodos não farmacológicos não são capazes para conquistar a cooperação e compreensão esperada do paciente. Por outro lado, o Cirurgião-dentista possui recursos farmacológicos que fazem o paciente atingir nível de sedação consciente desejado.

Os Benzodiazepínicos (BZDs) são descritos na literatura como fármacos de primeira escolha para o controle da ansiedade em consultório odontológico apresentando boa eficácia, propriedades ansiolíticas, sedativas, miorrelaxantes e anticonvulsivantes ${ }^{16}$. A sedação consciente por via oral com benzodiazepínicos apresenta baixa incidência de reações adversas, baixo custo e facilidade de administração ${ }^{17}$. Já para Oliveira ${ }^{16}$, a técnica de sedação consciente com $\mathrm{N} 2 \mathrm{O}$ com $\mathrm{O} 2$ é usada como principal sedação proporcionando um efeito relaxante para controle da ansiedade com efeitos analgésicos/sedativos e não pelo efeito anestésico. As principais vantagens dessa técnica em correlação com a sedação com BZD administrados por via oral são o tempo curto para alcançar os níveis adequados de sedação (por volta de 5 minutos) e recuperação dos efeitos sedativos onde que o paciente pode ser liberado do atendimento odontológico sem a necessidade de acompanhante ${ }^{18}$.

Oliveira $^{16}$ afirma que o Diazepam é considerado o fármaco padrão e o mais utilizado em procedimentos ambulatoriais que necessitam uma sedação pós-operatória mais prolongada e o Midazolam é a droga de escolha usada em pacientes adultos e pediátricos, por possuir rápido início de ação.
No quadro 5 evidencia-se que quanto a pergunta sobre a contribuição da sedação para um posterior condicionamento, todos os entrevistados responderam que acreditam que a sedação seja eficiente para contribuir com um posterior condicionamento dos pacientes, tendo em vista que não haverá lembranças negativas e possíveis traumas e em atendimentos anteriores sem o uso da sedação foi uma experiência negativa e traumática.

Conforme análise do quadro 6 , deixou-se em aberto alguma contribuição que os entrevistados gostariam de acrescentar sobre o uso da sedação. E, mais uma vez, as respostas, ainda que distintas, foram todas sinalizando de forma positiva e eficaz o uso da sedação em atendimento odontológico em pacientes com TEA.

CONCLUSÃO

As mães e pais de crianças diagnosticadas com TEA, demonstraram-se satisfeitos, sendo sua satisfação relacionada ao fato de terem encontrado na sedação, as características desejáveis a um atendimento seguro, eficaz,e sem traumas, aliadas à possibilidade de um posterior condicionamento, promovendo assim, um atendimento odontológico confortável.

A percepção dos pais de crianças diagnosticadas com Transtorno do Espectro Autista sobre o uso da sedação leve à moderada em atendimento odontológico tornase importante, por fornecer informações não só sobre o uso da sedação, mas também sobre a qualidade no atendimento que a sua utilização proporciona, bem como, a segurança da técnica, tranquilizando assim, outras famílias quanto ao seu uso.

REFERÊNCIAS

1. American PsychiatryAssociation (APA).(2014). Manual diagnóstico e estatístico de transtornos mentais-DSM-V. Porto Alegre: Artmed.

2. Oliveira KG, Sertié AL. Transtornos do espectro autista: um guia atualizadopara aconselhamento genético. Einstein. 2017;15: 233-38.

3. Gomes KAS, Vieira LDS, Ferreira RB. Autismo: uma abordagem comportamental. 2020.

4. Brito AR, Vasconcelos MM. Conversando sobre autismo - reconhecimento precoce $e$ possibilidades terapêuticas. In: Caminha V.L.; Huguenin JY, Assis LM, Alves PP. Autismo: Vivências e Caminhos. Blucher. 2016; 23-32.

5. Onzi FZ, Gomes RF. Transtorno do Espectro Autista: a importância do diagnóstico e reabilitação. Caderno pedagógico. 2015;12: 188-99. 
6. Sant'anna LFC, Barbosa, CCN, Brum SC. Atenção à saúde bucal do paciente autista. Rev Pró-UniverSUS. 2017;8:67-74.

7. Orellana LM, Silvestre FJ, Martínez-Sanchis S, Martínez-Mihi V, Bautista D. Oral manifestations in a group of adults with autism spectrum disorder. Med Oral Patol Oral Cir Bucal. 2012;17(3):e415-19.

8. Cagetti MG, Mastroberardino S. Campus G, Olivari B, Fagiolli $R$, Lenti $C$. Dental care protocol based on visual supports for children with autism spectrum disorders. Med Oral Patol Oral Cir Bucal. 2015;20(5):e598-604

9. Delli K, Reichart PA, Bornstein MM, Livas C. Management of children with autism spectrum disorder in the dental setting: Concerns, behavioural approaches and recommendations. Medicina Oral, Patología Oral y Cirugía Bucal. 2013;18(6):e862-68.

10. Lemos JPC. Caracterização dos pacientes com diagnóstico de Transtorno do Espectro Autista atendidos no Núcleo de Odontologia Hospitalar do Hospital Universitário Professor Polydoro Ernani de São Thiago - HU UFSC[monografia]. Florianópolis: Faculdade de Odontologia, Universidade Federal de Santa Catarina; 2017.

11. Polli VA, Sordi MB, Lisboa ML, Munhoz EA, Rodigues $A$ de $C$. Dental management of special needs patients: a literature review. Global J Oral Sci. 2016;2:33-45.

12. Araujo LC, Romero B. Dor: avaliação do $5^{\circ}$ sinal vital. Uma reflexão teórica. Rev dor. 2015;16(4):291-96.

13. Jordan KP, Sim J, Croft P, Blyth F. Pain that does not interfere with daily life-a new focus for population epidemiology and public health? Pain. 2019;160(2):281-85.

14. Berkovits L, Eisenhower A, Blacher J. Emotion Regulation in Young Children with Autism Spectrum Disorders.J Autism Dev Disord. 2017;47:68-79.

15. Oliveira BF. Sedação na odontologia em pacientes com necessidades especiais: revisão de literatura [monografia]. Uberaba: Curso de Odontologia, Universidade de Uberaba; 2018.

16. Rang HP, Dale MM, Ritter JM, Flower RJ. Farmacologia. 6. ed. Rio de Janeiro: Elsevier; 2017;535-44.

17. Glassman P, Caputo A, Dougherty N, Lyons R, Messieha Z, Miller C et al. Special care dentistry association consensus statement on sedation, anesthesia, and alternative techniques for people with special needs. Spec Care Dentist. 2009;29(1):2-8.

18. Dougherty $\mathrm{N}$. The dental patient with special needs: a review of indications for treatment under general anesthesia. Spec Care Dentist. 2009;29(1):17-20.

\section{CONFLITO DE INTERESSES}

Os autores declaram não haver conflitos de interesse

AUTOR PARA CORRESPONDÊNCIA

Herrison Félix Valeriano da Silva

Universidade Federal da Paraíba (UFPB)

58051-900 João Pessoa - PB, Brasil

E-mail: herrison.felix.vds@gmail.com

Submetido em 28/07/2021

Aceito em 18/10/2021 